\title{
P04-1-46 Poster session
}

\section{AMPK activation by $\mathrm{P} 2 \mathrm{X7}$ through ROS-CaMKK pathway regulates mitophagy and lysosomal biogenesis in macrophages}

\author{
Ponarulselvam Sekar ${ }^{1}$, Duenyi Huang ${ }^{2}$, Wan-Wan Lin ${ }^{1,2}$ \\ ${ }^{1}$ Graduate institute of medical sciences, Taipei Medical university, Taipei, Taiwan, ${ }^{2}$ Graduate institute of \\ pharamacology, National taiwan university, Taipei, Taiwan
}

Background: Mitophagy is the selective engulfment of damaged mitochondria by autophagosomes and their subsequent catabolism by lysosomes, and majorly it has been considered to prevent neurodegeneration diseases. Accumulating findings reveal AMPK-associated mitophagy as a means to cope with energy and nutritional stress. Since mitochondrial function in microglia is highly associated with microglial functions in controlling neuronal plasticity and brain homeostasis, we interested to explore the roles of P2X7, a danger signal receptor, in mitochondrial and lysosomal functions in microglia.

Methods: In microglial cells and bone marrow derived macrophages (BMDM), we used BzATP for the activation of P2X7. To determine the P2X7-induced mitophagy and lysosomal biogenesis, we adopted some tracker dyes like mitotracker green and lysotracker red using flow cytometry. For further confirming the mitochondrial fragmentation and mitochondrial respiration, we used confocal microscopy and seahorse XF analysis.

Results: P2X7 activation triggered AMPK activation and LC3II accumulation through ROS and CaMKKII pathways, and these effects were abolished by P2X7 antagonist and P2X7 knockout. Moreover, AMPK inhibition can reverse the P2X7 action on reduction of mitochondrial mass as indexed by the Mitotracker Green staining. Confocal microscopic images indicate the induction of mitochondrial fission and mitophagy after P2X7 activation and the significant blockade of these effects by AMPK inhibition. To mimic the actions of P2X7, we used nigericin to explore the above signaling mechanisms. As a result, we found the ability of nigericin to induce ROS-dependent AMPK activation and mitophagy. Interestingly, P2X7 activation can induce nuclear translocation of TFEB via AMPK dependent pathway, increase lysosomal rupture and cathepsin B release. Moreover, autophagic and cathepsin B inhibitors can reduce BzATP-induced cell death.

Conclusion: Altogether, ROS- and CaMKK-dependent AMPK activation is involved in P2X7-mediated mitophagy, mitochondrial fission and lysosomal biogenesis in microglia, which may play an important role in inflammation associated disease progression. 